\title{
Research Methods in the Field of Computer Visualization Based on Virtual Reality
}

\author{
Natalya Averbukh ${ }^{1}[0000000282326711]$ and Vladimir Averbukh ${ }^{1,2}[0000000243791450]$ \\ ${ }^{1}$ Ural Federal University Yekaterinburg, Russia natalya_averbukh@mail.ru \\ ${ }^{2}$ Krasovskii Institute of Mathematics and Mechanics, Yekaterinburg, Russia \\ averbukh@imm.uran.ru
}

\begin{abstract}
The paper examines research methods in the field of computer visualization based on virtual reality. Developing such systems involves not only the issues of software implementation, modelling or adequate equipment choice, but also the tasks connected with cognitive processes occurring in both users and developers of visualization systems. Featured is a brief review of papers dealing with research in human interaction with virtual reality. The scope of tasks in developing visualization systems based on virtual reality is determined. The conclusion discusses several research issues, the solutions to which will increase the efficiency of interaction of users with virtual environments.
\end{abstract}

Keywords: virtual reality $\cdot$ computer visualization $\cdot$ mental model $\cdot$ cognitive processes.

\section{Introduction}

Virtual reality environments were initially used for training simulation, but as far back as in the late 1980s scientific visualization systems based on virtual reality occurred. Those were the pilot versions of virtual test stands that were used in developing reusable space shuttles [6]. Currently, virtual reality environments have been actively used in scientific visualization and software visualization systems.

Developing such systems involves not only the issues of software implementation, modelling or adequate equipment choice, but also the tasks connected with cognitive processes occurring in both users and developers of visualization systems. There also occurs the problem of perception of spatial characteristics: depth and distance, absolute and relative dimensions of objects, distance to them. This problem occurs due to the fact that when navigating in virtual reality, especially when using the so called natural interfaces and 'direct interaction' with displayed objects, a user, naturally, should understand the distance to them and between them. Apart from that, the issue of perception of spatial characteristics can be related to the information, transmitted by the object's characteristics, if any abstract parameters are coded with object dimensions, their correlation, distance between them or from them to a certain point. Thus, developing computer visualization systems demands research in the field of computer psychology.

Copyright $($ C 2020 for this paper by its authors. Use permitted under Creative Commons License Attribution 4.0 International (CC BY 4.0). 


\section{N. Averbukh and V. Averbukh}

\section{Related works}

A virtual environment can act both as an instrument of research, in the field of psychology in particular, and as an object of research. To be more precise, the interaction of a person with virtual reality is an object of research, alongside with those special aspects that manifest themselves in a person's behavior in a virtual environment. Another important field is the research into the potential of using virtual environments as the basis for computer visualization systems. A whole range of papers are devoted to these issues. The papers of Slater's group discuss both the issues of measuring states specific to virtual reality [16], [20], [17], [18] and the experience of measuring a person's notion of their own body in the process of using an avatar in virtual reality [19], [9], [4], [10], [8], which can be applied in such a direction as immersive journalism, when a person is offered to 'personally experience' the events covered by the mass media[21].

In our country, the interconnection between the state of presence, specific to virtual reality, and cognitive control (i.e. a metacognitive function controlling the accuracy of fulfilling a certain task) is established [22].

There are also tasks connected with the perception of depth in virtual reality, see further [12] and [15].

Research into the potential of virtual reality is also featured in the papers devoted to evaluating the efficiency of 3D software visualization systems based on virtual reality, when using a city metaphor, such as [7]. The efficiency of visualization is evaluated in terms of performance, a range of impressions and user experience.

Two types of visualization are typically discussed in academic research: illustrative visualization and cognitive visualization. Illustrative visualization demonstrates the already known phenomena, while cognitive visualization facilitates cogitation by showing brand new effects that provide new knowledge [23]. Apart from that, one can distinguish demonstrative visualization, which shows the results of quantitative research, thus proving (or refuting) certain ideas of a mathematician (or any other expert working with the visualization system). Three types of imagery are used in this case: natural imagery, reflecting real features of the model; imagery traditional to the given application domain (physics, chemistry, biology, mathematics etc.); and abstract imagery, which should be invented for any specific case, perhaps, based on visualization metaphors basic perception ideas for visual objects [3].

\section{Determining the tasks for the development of visualization systems based on virtual reality}

It is worth noting that experts setting the tasks of visualizing certain objects, processes or phenomena have specific ideas of their structure that can be called mental models. Situations may occur when an analyst does not know what the visualization should look like, but he or she knows exactly what it does not look like. At the same time, he or she is not always able to describe it clearly. A visualization developer should also have the idea of a mental model of the object, process or phenomenon. That is why he or she should have sufficient knowledge in the specific field and should grasp the essence of the simulation task thoroughly. Nevertheless, an analyst often sees much 
more in the resulting 'picture', than the developer has expected them to. For example, when visualizing one of the tasks of mathematical physics dealing with air condition around a flying object an analyst 'saw' much more characteristics than the developers had expected. It turned out that, by using color shades to express pressure, temperature etc., they managed to convey the intermediate values, which gave additional information to the expert.

The work of a client and a visualization system user is largely explained in Brushlinskiy's papers. According to him, the unknown is not some kind of an 'absolute void', which cannot be operated with. It exists in a certain system of interrelations linking it with what is already given in the problem. It is in the course of disclosing these interrelations that something new can be extracted [5]. Brushlinskiy describes cogitation as a process where the solution is anticipated at the first stage, gradually unfolding at further stages of the cognitive process. Thus, a visual analyst already foresees the way visual objects reflecting the model should look at the discussion stage.

The users of visualization systems based on virtual reality environments get information additional to that acquired in the process of using traditional visualization methods. Moving around a virtual space allows the user to see the inside of complex objects and their interrelation.

When using virtual reality, in order to ensure its advantages in visualization one should develop special views that enable factoring these advantages in. In the case of software visualization, various visualization metaphors exist, which can be used to create such views; for instance, a popular city / landscape metaphor.

In the case of scientific visualization, one should consider each situation separately, even if traditional methods of data representation should be used; for example, in the case of computational grids visualization, when it is clear what to show but not entirely clear how to do it in order to ensure the advantages of virtual reality environments or the expert's sustainable work process. Therefore, one should take into consideration the distortions that occur during interaction with virtual reality.

Developed views should comply with the models of phenomena, processes and objects, as well as with users' mental models. They should ensure those advantages that virtual reality offers.

As noted above, a developer should study the application domain, work in close cooperation with analysts/users in order to take into consideration their mental models and carefully examine the features of implementing visualization systems based on virtual reality and interface interaction in such systems.

When developing views for systems based on virtual reality one should also take into consideration the role of interfaces. Two types of interfaces can be distinguished in the systems based on virtual reality: one for controlling and adjusting the system itself and the virtual reality, and one for working directly with the model. These interfaces can be of radically different types. For instance, one interface can be natural, with the use of gestures or voice commands [11]; while the other one can use various devices such as a keyboard, a joystick etc.

In the process of researching human interaction with virtual reality systems, an issue of studying the human factor inevitably arises. Firstly, one can distinguish the aforementioned issue of distortions in the perception of space, depth and distances in virtual 
reality. Interesting work has been done before in the field of depth perception in virtual reality environments, which involved demonstration of visual feedback value when reaching a virtual object [12]. The role of a familiar dimension of the object the user was offered to reach was also examined when tackling the same task [15]. Apart from that, [15] shows that a task is better fulfilled if the object that the user is offered to reach is specific, not an abstract one. Although an abstract object rather means a 'simple box', while in visualization systems, this typically refers to complex structured objects with high semantic charge. However, it is possible that the aforementioned principle will also relate to them, as even such objects are unfamiliar to the user in a trivial way, are not present in their experience and, therefore, do not have a familiar dimension.

The issue of accuracy in reaching a virtual object may turn out critical in using natural interfaces. Another issue, connected with spatial characteristics, is the issue of transmitting certain features of the simulated object by means of length, width, height and depth, as well as overall volume or area of certain surfaces. Research is required into whether it is possible to adequately perceive dimensional features of virtual objects by interacting with them.

Of importance are also aspects connected with cognitive processes and aspects dealing with the psychomotor system, which is inevitably involved when interacting with a visualization system based on virtual reality. One should not forget that the issue of proprioceptive feedback in virtual reality remains unsolved, that is why all movements in virtual reality are perceived differently in comparison with the physical world.

As is shown by individual examples in [2], in virtual reality, interaction with the controller can substitute natural interaction, and a person may reflexively push the button in situations where he or she would clench their fists or grab something in the real world.

As demonstrated in [2], being in a virtual reality requires simultaneous perception of both the real space and the virtual space from the person switching between them [17]. This may influence the interaction with the model or the decisions made by the person. Apart from the issues mentioned above, the questions relating to the choice between realism (which is more easily perceived) and abstraction (which may be more adequate for the model) also arise when developing visualization systems based on virtual reality. This issue also includes the problem of developing interfaces used in visualization systems based on virtual reality. As mentioned before, interaction with realistic objects, which have familiar dimensions, is more convenient for the user because they can calculate their movements more easily than in the case of interaction with abstract objects. Hence, the choice of visualization metaphors and interface.

\section{Conclusion. Setting research problems}

Developing visualization systems based on virtual reality sets a whole range of research tasks connected with the influence of spatial perception on the interaction with models in visualization systems or dealing with the psychomotor system.

These could be tasks relating to object dimension evaluation. The difference from conventional spatial perception experiments is in the fact that only at the first stage can the experimental task involve purely evaluating the dimensions. The main part of 
such research should involve evaluation of parameters displayed by means of linear dimensions, surface area or volumes, both absolute and relative.

Another issue related to space deals with a person's ability to find their way in a virtual world. Experience shows that, in some cases, a person moving around in a complex virtual scene, which is impossible to scan at once, does not memorize the route they have 'travelled'. This may turn out critical in using a landscape metaphor or a city metaphor.

On the other hand, we should examine the efficiency of various interface principles (the natural ones and the 'device' ones), their convenience for users and their applicability for various tasks occurring in visualization systems based on virtual reality. For instance, the papers [14] and [13] offer interfaces that enable the use of a manipulator glove detecting spatial gestures of the person [14] or interaction with tangible exhibits of a paleontology museum [13].

The issues of evaluating the potential of solving complex intellectual tasks in virtual reality also remain relevant. Such issues have been offered solutions, for example, in [1], but the topic requires further elaboration.

\section{References}

1. Averbukh, N., Shcherbinin, A.: The presence phenomenon and its influence upon intellectual task performance within virtual reality settings. Psychology. Journal of the Higher School of Economics 8(4), 102-119 (2011), in Russian

2. Averbukh, N.V.: On the issue of perception of space when experiencing the phenomenon of presence in virtual reality. In: Ershova, R.V. (ed.) Digital society as a cultural and historical context of human development: a collection of scientific articles. pp. 11-15. State social and humanitarian University, Kolomna (2020), in Russian

3. Averbukh, V.: Visualization metaphors. Programming and Computer Software 27(5), 227237 (September 2001)

4. Banakou, D., Groten, R., Slater, M.: Illusory ownership of a virtual child body causes overestimation of object sizes and implicit attitude changes. Proceedings of the National Academy of Sciences of the United States of America 110(31), 12846-12851 (2013)

5. Brushlinskiy, A.: Actor, Thinking, Learning, Imagination. MPSI, Moscow (2003), in Russian

6. Bryson, S.: Virtual environments in scientific visualization. In: VRST ' 94 Proceedings of the conference on Virtual reality software and technology. pp. 201-220 (1994)

7. Merino, L., Fuchs, J., Blumenschein, M., Anslow, C., Ghafari, M., Nierstrasz, O., Behrisch, M., Keim, D.: On the impact of the medium in the effectiveness of $3 \mathrm{~d}$ software visualizations. In: Proceedings on 2017 IEEE Working Conference on Software Visualization (VISSOFT). pp. 11-21 (2017)

8. Neyret, S., Bellido Rivas, A., Navarro, X., Slater, M.: Which body would you like to have? the impact of embodied perspective on body perception and body evaluation in immersive virtual reality. Frontiers in Robotics and AI 7(31) (2020). https://doi.org/10.3389/frobt.2020.00031

9. Normand, J.M., Giannopoulos, E., Spanlang, B., Slater, M., Giurfa, M.: Multisensory stimulation can induce an illusion of larger belly size in immersive virtual reality. PLoS ONE 6(1), e16128 (2010). https://doi.org/110.1371/journal.pone.0016128

10. Peck, T., Seinfeld, S., Aglioti, M., Slater, M.: Putting yourself in the skin of a black avatar reduces implicit racial bias. Consciousness and Cognition 22(3), 779-787 (September 2013) 
11. Pestova, M., Starodubtsev, I.: Development of specialized gesture interfaces for scientific visualization systems. In: GraphiCon 2016: Proceedings of the International scientific conference. pp. 369-373. Nizhny Novgorod (September 19-23 2016), in Russian

12. Renner, R.S., Steindecker, E., MüLler, M., Velichkovsky, B.M., Stelzer, R., Pannasch, S., Helmert, J.R.: The influence of the stereo base on blind and sighted reaches in a virtual environment. ACM Trans. Appl. Percept. 12(2) (2015). https://doi.org/10.1145/2724716, https://doi.org/10.1145/2724716

13. Ryabinin, K., Akhtamzyan, A., Kolesnik, M., Sudarikova, E.: Tangible interfaces for the virtual reconstructions of museum exhibits. In: GraphiCon 2019: Proceedings of the International scientific conference. vol. 1, pp. 8792. Bryansk, Russia (2020). https://doi.org/10.30987/graphicon-2019-1-87-92, https://vestnik.astu.org/ru/nauka/conference_article/3665/view, in Russian

14. Ryabinin, K., Belousov, K., Chuprina, S., Zelyanskaya, N.: Perceptivecognitive user interface for visual analytics systems. In: GraphiCon 2019: Proceedings of the International scientific conference. vol. 1, pp. 93-98. Bryansk, Russia (2020). https://doi.org/10.30987/graphicon-2019-1-93-98, https://vestnik.astu.org/ru/nauka/conference_article/3666/view, in Russian

15. Schubert, R.S., Müller, M., Pannasch, S., Helmert, J.R.: Depth information from binocular disparity and familiar size is combined when reaching towards virtual objects. In: VRST '16 (2016)

16. Slater, M.: Measuring presence: A response to the witmer and singer presence questionnaire. Presence, Teleoperators and Virtual Environments 8, 560-565 (October 1999)

17. Slater, M.: Presence and the sixth sense. Presence 11(4), 435-439 (August 2002)

18. Slater, M.: Place illusion and plausibility can lead to realistic behaviour in immersive virtual environments. Philosophical Transaction of Royal Society B: Biological Sciences 364, 35493557 (2009)

19. Slater, M., Spanlang, B., Sanchez-Vives, M.V., Blanke, O.: First person experience of body transfer in virtual reality. PLoS ONE 5(5), e10564-e10564 (2010). https://doi.org/10.1371/journal.pone.0010564

20. Slater, M., Steed, A.: A virtual presence counter. Presence, Teleoperators and Virtual Environments 9(5), 413-434 (2000)

21. Steed, A., Pan, Y., Watson, Z., Slater, M.: "we wait"- the impact of character responsiveness and self embodiment on presence and interest in an immersive news experience. Front. Robot. AI (2018)

22. Velichkovsky, B., Gusev, A., Kremlev, A., Grigorovich, S.: Cognitive control influences the sense of presence in virtual environments with different immersion levels. Lecture Notes in Computer Science 10324, 3-16 (2017)

23. Zenkin, A.: Cognitive Computer Graphics. Applications in Classical Theory of Natural Numbers. (Synopsis). "NAUKA', Moscow (1991), in Russian 